\title{
EDITORIAL
}

\section{Human capital in translational research}

\author{
The past two years have seen the launch of a range of high-profile initiatives to encourage \\ translational research. A key to their long-term success is creating an environment in which \\ investigators in this pivotal discipline have a clearer career path.
}

In 2006, the US National Institutes of Health (NIH) launched the Clinical and Translational Science Awards (CTSA) programme - a major part of its ongoing effort to accelerate the translation of biomedical research into therapeutic applications. The aim: to develop a consortium of institutes to transform clinical and translational research in the United States.

Two years on, the programme currently funds 24 academic medical centres, and is planned to grow to support approximately 60 by 2012, with an annual budget of $\sim$ US $\$ 500$ million. Initiatives elsewhere with similar goals are also gaining momentum. For example, the UK's National Institute for Health Research (NIHR) recently announced 12 new biomedical research units linking hospitals and academic centres to focus on translational research, which will together receive approximately $£ 45$ million over the next 4 years.

Nevertheless, this influx of funding, although highly welcome, will not on its own ensure that the goals of translational research are effectively achieved. Other key issues in this respect were among the topics discussed at the third of a series of symposia organized by the Institute for Translational Medicine and Therapeutics in Philadelphia, USA, and supported by Nature Reviews Drug Discovery (see http://www.itmat.upenn.edu).

One prominent factor, which is also highlighted in the news story on page 463 of this issue, was the researchers at centres who receive the funding. There has long been an awareness in the drug discovery and development community that people with key skills for translational research - for example, in in vivo pharmacology - had been becoming rarer. So, an important first step when grants dedicated to translational research started to become more widely available was to attract a new generation of students to consider what was then a relatively unconventional career starting point.

In this respect, initial reports from the United States suggest significant success. For example, a number of academic medical centres have noted a surge in interest in new masters programmes in clinical and translational science, tailored to cater for both physicans and research scientists. Through such programmes, basic researchers gain understanding of how physicians think about their patient's problems, and physicians learn techniques for investigating clinical observations in the laboratory. As a consequence, a growing number of well-trained translational investigators are poised to emerge.

However, the options that are available for them when they do emerge are at present much less clear than would be desirable. For a typical researcher with a Ph.D. and postdoctoral experience in biomedical sciences, the step to becoming an independent investigator is a challenging one. But for a young scientist trained in translational research, this challenge is further compounded by the nature of the discipline.

First, there is the well-acknowledged problem of recognition of achievement in translational research. For a biomedical researcher hoping to receive an independent grant, a crucial factor in their application would be their publication record, especially of high-profile papers to which they made a major contribution. However, translational research is inherently multidisciplinary, making it harder to recognize individual efforts. In addition, translational research seems to have struggled so far to gain the same profile in major journals as hot topics in biology, for example.

Second, there is the time needed to discover something meaningful in a translational research project. Even before a project can begin, the logistics of studies involving patients can require significant time, effort and expertise; for example, to satisfy the requirements of institutional review boards overseeing clinical trials. And many such studies could take months or even years before results are available - a sharp contrast with the pace of some hot areas of biological research.

Given such challenges, there is a clear need to better define a career path for the new generation of scientists seeking to pursue translational research. Ensuring that the current level of investment is at least maintained is important, but perhaps even more vital is recognizing what constitutes success for a researcher in this bridging discipline. As Joseph McCune, director of a CTSA-funded centre at the University of California, San Francisco, highlights on page 464: "If they don't know the rules for success, then they won't believe they can succeed". 